\title{
Septic Arthritis Due to Staphylococcus Warneri: A Diagnostic Challenge
}

\author{
Barbara Legius, Kristel Van Landuyt, Patrick Verschueren and Rene Westhovens*
}

Division of Rheumatology, University Hospital KU Leuven, Belgium

\begin{abstract}
A septic arthritis due to an indolent infection is a challenge for timely diagnosis. In recent years septic arthritides due to Staphylococcus Warneri are increasingly reported, mostly as a complication in patients with prosthetic devices. We report on a case of a 38 year old immunocompetent male with an indolent infection with this commensal of the skin after a stay at an intensive care unit and review the available literature. Tissue cultures obtained by arthroscopy might be helpful in obtaining a correct diagnosis.
\end{abstract}

Keywords: Septic arthritis, Staphylococcus Warneri, needle arthroscopy.

\section{INTRODUCTION}

Septic arthritis is usually seen as an acute monoarthritis leading to rapid joint destruction without proper and timely treatment. More indolent infections however often escape timely diagnosis. We report on a case of a 38 year old immunocompetent male patient with an indolent septic arthritis due to Staphylococcus Warneri. The current sparse literature on infections with this commensal of the skin is reviewed with a special focus on prosthetic device infections. There is always a risk of false diagnoses of infections with commensals because of sample contamination, but tissue cultures can be very helpful in obtaining a correct diagnosis.

\section{CASE REPORT}

The patient was referred with a 6 month history of a warm, swollen and painful right knee. Initially there was redness, which resolved spontaneously. First symptoms occurred 10 weeks after a car accident which resulted in a craniocerebral trauma, with coma and intracranial hypertension, requiring temporary ventricular drainage. During ICU stay, he developed a sepsis with Klebsiella Pneumoniae and Pseudomonas Aeruginosa and there was a period of multi-organ failure. Swelling of the right knee was only noticed at the start of rehabilitation and no evidence of infection was found in the synovial fluid. There was no knee trauma reported at the time of the car accident nor during hospital stay. He was referred for further diagnosis. Patient consented orally for anonymous publication of this case report.

Clinical examination at presentation revealed a warm, swollen, painful right knee, without redness. There were no other joints involved. Body temperature was normal. The remainder of the clinical examination was normal. Arthrocentesis of the knee showed a white blood cell count (WBC) of 57000 with $90 \%$ neutrophils. Cultures were again

*Address correspondence to this author at the Herestraat 49, 3000 Leuven Belgium; Tel: +32-16-342541; Fax: +32-16-342543;

E-mail: rene.westhovens@uzleuven.be negative, including a mycobacterial culture. There were no crystals in the fluid. Laboratory tests showed an elevated Creactive protein (CRP $43 \mathrm{mg} / \mathrm{L})$ and elevated blood WBC of $11.500 / \mathrm{mm}^{3}$ ). Rheumatoid factor, anti-CCP antibodies and anti-nuclear factor were negative as were Borellia antibodies, Chlamydia Trachomatis PCR and Neisseria Gonorrhoeae PCR. Magnetic resonance imaging of the knee showed no major cartilage problems and no meniscal or ligamental lesions but several bony infarctions in femur and tibia. For further diagnosis a needle arthroscopy was performed revealing a hyperemic and very hypertrophic synovium covered with a white film (See supplementary file). Biopsies of synovial tissue were obtained and showed important inflammation with a relatively high amount of neutrophils. Direct cultures of the synovial tissue showed staphylococcus Warneri, resistant only to penicillin. PCR for Borrelia, mycobacteria genus and mycobacterium complex on the biopsy specimen were negative. Treatment with Levofloxacin was initiated for 6 weeks. Clinical evolution showed clear improvement although with some persisting swelling of the knee after 6 weeks of antibiotic treatment. Because of the mild persisting gonartritis at that time a needle arthroscopy was repeated. Synovial biopsies showed a decreased inflammatory infiltrate and the synovial fluid now only revealed 3.810 WBC with $38 \%$ neutrophils compared with $78.100 \mathrm{WBC}$ and $90 \%$ neutrophils at the time of first arthroscopy just before starting therapy. CRP dropped from 66.2 at the moment of first arthroscopy to 26.2 $\mathrm{mg} / \mathrm{l}$ after 6 weeks of treatment at the time of the second arthroscopy.

\section{DISCUSSION}

Staphylococcus Warneri is a coagulase-negative staphylococcus. It is a commensal of the skin, found in $50 \%$ of the population. This species usually represents less than $1 \%$ of the total staphylococcal population [1]. Coagulasenegative staphylococci have become common nosocomial pathogens [2]. They are an etiologic agent of infections of prosthetic devices [3]. A few cases of S. Warneri infections have been reported in the literature. Septicaemia, endocarditis, discitis, osteomyelitis, subdural empyema and urinary tract infections have been described [4,5]. So far, 7 
cases of S. Warneri endocarditis have been reported [2], as have been 4 cases of S. Warneridiscitis [4]. In a series of orthopedic implant infections, Arciola et al. found $\mathrm{S}$. Warneri in $2.6 \%$ of the cases [6,7]. Campoccia et al. described articular infections with S. Warneri in the absence of prosthetic material S. Warneri related infections have also been reported in neonates [8]. Cimiotti et al. found that S. Warneri can be pathogenic in neonates. Out of 647 bacterial isolates associated with clinical infection in neonates in a neonatal intensive care unit, 17 infections were caused by S. Warneri, including 15 bloodstream infections, 1 skin infection and 1 eye infection [8].

However, coagulase negative staphylococci, such as S. Warneri, as commensals of the skin, areconsidered frequent contaminants of blood cultures as well [1]. Eng et al. reported a series of 142 patients with coagulase negative staphylococci positive blood cultures. In 96 patients only one single blood culture bottle revealed staphylococci and in these patients the presence of coagulase negative staphylococci was therefor considered as a contamination [9]. In clinical practice therefore the diagnosis of a $\mathrm{S}$. Warneri infection will depend on the weight of the evidence that this commensal could indeed be responsible for the symptoms and signs under consideration.

In this case report, we report an articular infection with S. Warneri without orthopedic implant. It is possible that S. Warneri entered the blood stream through a catheter during an admission at an ICU, 10 weeks before onset of symptoms. $\mathrm{S}$. Warneri is known to be of low virulence and grow slowly [4]. This might explain the delay between exposure of $\mathrm{S}$. Warneri and onset of symptoms. Because of negative cultures of articular fluid there was a considerable delay to diagnosis. Cultures of synovial tissue, obtained by arthroscopy, revealed the causative agent as being a Staphylococcal Warneri. This case is instructive because it emphasizes the importance of tissue sampling in arthritis of unknown origin. In the absence of positive articular fluid cultures, an arthroscopic intervention with collection of synovial biopsies can lead to a diagnosis.

Culture of tissue obtained by surgical intervention might be important in other infections of unknown etiology as well. Abgrall et al. reports a prosthetic valve endocarditis due to $\mathrm{S}$. Warneri, with a lack of positive blood cultures. Culture of vegetation tags of the surgically removed prosthetic valve revealed a Staphylococcus Warneri [10].

\section{CONCLUSION}

Staphylococcus Warneri is a coagulase negative staphylococcus that infrequently causes infections of prosthetic devices [3]. Diagnosis can be challenging due to low virulence and slow growth [4]. We present a case of arthritis of the knee due to S. Warneri, in the absence of a prosthetic device. Diagnosis was established by culture of arthroscopically obtained synovial tissue. This case is instructive because it emphasizes the importance of tissue sampling in arthritis of unknown origin. In the absence of positive articular fluid cultures, arthroscopic intervention can lead to a diagnosis.

\section{CONFLICT OF INTEREST}

The authors confirm that this article content has no conflicts of interest.

\section{ACKNOWLEDGEMENT}

Declared none.

\section{SUPPLEMENTARY MATERIAL}

Supplementary material is available on the publisher's web site along with the published article.

\section{REFERENCES}

[1] Kloos W. Natural populations of the genus Staphylococcus. Ann Rev Microbial 1980; 34: 559-92.

[2] Arslan F, Saltoglu N, Mete B, Mert A. Recurrent Staphylococcus Warneri prosthetic valve endocarditis: A case report and review. Ann Clin Microbiol Antimicrob 2011; 10: 14.

[3] Wood CA, Sewell DL, Strausbaugh LJ. Vertebral osteomyelitis and native valve endocarditis caused by Staphylococcus warneri. Diagn Microbiol Infect Dis 1989; 12: 261-3.

[4] Announ N, Mattei JP, Jaoua S, et al. Multifocal discitis caused by Staphylococcus warneri. Joint Bone Spine 2004; 71: 240-2.

[5] StöllbergerC, Wechsler-Fördös A, Geppert F, et al. Staphylococcus warneri endocarditis after implantation of a lumbar disc prosthesis in an immunocompetent patient. J Infect 2006; 52: e15-8.

[6] ArciolaCR, Campoccia D, An YH, et al. Prevalence and antibiotic resistance of 15 minor staphylococcal species colonizing orthopedic implants. Int J Artif Organs 2006; 29: 395-401.

[7] CampocciaD, Montanaro L, Visai L, et al. Characterization of 26 Staphylococcus warneri isolates from orthopedic infections. Int J Artif Organs 2010; 33: 575-81.

[8] CimiottiJP, Haas JP, Della-Latta P, Wu F, Saiman L, Larson EL. Prevalence and clinical relevance of Staphylococcus warneri in the neonatal intensive care init. Infect Control Hosp Epidemiol 2007; 28: 326-30.

[9] EngRH, Wang C, Person A, Kiehn TE, Armstrong D. Species Identification of coagulase-negative staphylococcal Isolates from blood cultures. J Clin Microbiol 1982; 15: 439-42.

[10] AbgrallS, Meimoun P, Buu-Hoi A, Couetil JP, Gutmann L, Mainardi JL. Early prosthetic valve endocarditis due to Staphylococcus warneri with negative blood culture. J Infect 2001; 42: 166-9. 